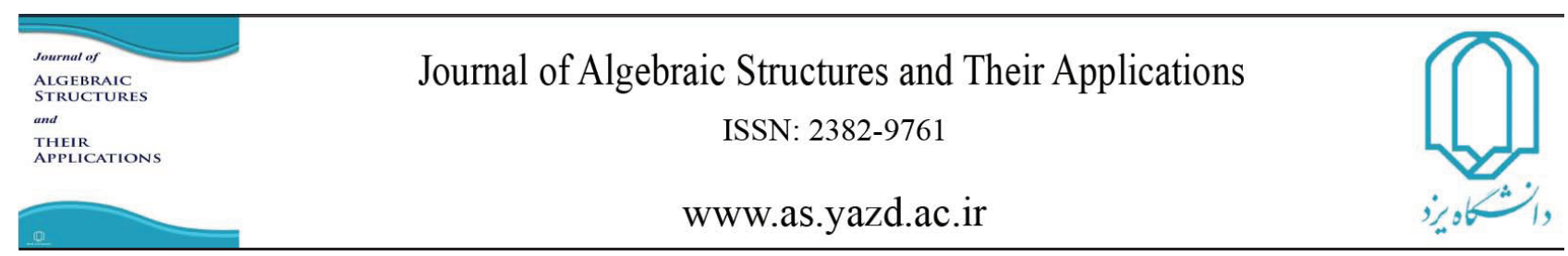

Algebraic Structures and Their Applications Vol. 6 No. 1 ( 2019 ) pp 1-21.

\title{
ROUGH IDEALS BASED ON IDEAL DETERMINED VARIETIES
}

\author{
SAEED RASOULI
}

\begin{abstract}
The paper is devoted to concern a relationship between rough set theory and universal algebra. Notions of lower and upper rough approximations on an algebraic structure induced by an ideal are introduced and some of their properties are studied. Also, notions of rough subalgebras and rough ideals with respect to an ideal of an algebraic structure, which is an extended notion of subalgebras and ideals in an algebraic structure, are introduced and investigated.
\end{abstract}

\section{INTRODUCTION}

Formal concept analysis (FCA) and rough set theory (RST), introduced in the early 80s by Wille [44] and Pawlak [28], respectively. These theories have become pioneering of knowledge acquisition from data tables. This fact has resulted in a rapid growth of interest in their formal relationships and possible unifications. Generally, both theories are based on Galois connections and cluster data into coherent and meaningful entities called concepts. These theories are useful tools for qualitative data analysis.

DOI : $10.29252 /$ as. 2019.1334

MSC(2010): 06F99,06D20

Keywords: rough set, approximation, universal algebra, subalgebra, rough subalgebra, ideal, rough ideal.

Received: 04 Dec 2018, Accepted: 14 Feb 2019

*Corresponding author

(C) 2019 Yazd University. 
The theory of rough set was first introduced as a tool for dealing with granularity in knowledge. Rough set theory is an extension of set theory. In the theory of rough set, it is important to construct a pair of upper and lower approximation operators based on available information. The Pawlak approximation operators are defined by an equivalence relation. The equivalence classes are the building blocks for the construction of the lower and upper approximations.

Formal concept analysis is formulated based on the notion of a formal context, which is a binary relation between a set of objects and a set of properties or attributes [15, 44]. The binary relation induces set-theoretic operators from sets of objects to sets of properties, and from sets of properties to sets of objects, respectively. A formal concept is defined as a pair of a set of objects and a set of properties connected by the two set-theoretic operators.

The notion of formal contexts provides a common framework for the study of rough set theory and formal concept analysis, if rough set theory is formulated based on two universes. Dntsch and Gediga pointed out that the set-theoretic operators used in the two theories have been considered in modal logics, and therefore referred to them as modal-style operators [16]. They have demonstrated that modal-style operators are useful in data analysis.

Some researches studied algebraic properties of rough sets. Iwinski [22] suggested a lattice theoretic approach to rough set. Bonikowaski [5] studied some algebraic and set-theoretical properties of rough sets. Pomykala [2.9] showed that the set of rough sets forms a Stone algebra. A natural question is what will happen if we substitute an algebraic structure instead of the universe set. Biswas and Nanda [3], applied the notion of rough sets to algebra and introduced the notion of rough subgroups. Kuroki and Wang [24] gave some properties of the lower and upper approximations with respect to the normal subgroups. Kuroki [25], introduced the notion of a rough ideal in a semigroup. Davvaz [7] studied the properties of rough subring with respect to ideals of ring. Rough modules [9] have been investigated by Davvaz and Mahdavipour. Rasouli and Davvaz [33], introduced and studied the notion of a rough ideal in an MV-algebra. Fuzzy rough sets were defined by Dubois and Prade [II]. Yao [47] introduced the concept of generalized rough sets based on relations. Feng et al. proposed another type of rough sets, in which lower and upper approximations of a subset are obtained with the help of soft sets [12], also, see [8, 201, 30, 31, 32, 34, 35, 45, 46].

In many of the familiar classes of algebras, congruences can be adequately represented by suitable subsets of the universes of the algebras. This is a desirable phenomenon that considerably simplifies the study of congruences and homomorphisms in such classes. In a group the representing sets are the normal subgroups of the group, in a ring the two-sided ideals, and in algebras of logic such as residuated lattices, divisible residuated lattices, MTLalgebras, BL-algebras, MV-algebras, Heyting algebras and Boolean algebras the lattice filters. The sets are characterized by certain closure properties which ensure that they coincide with 
the congruence classes containing a given constant - the group identity in the case of groups and rings, the top element in that of residuated lattices, divisible residuated lattices, MTL-algebras, BL-algebras, MV-algebras, Heyting algebras and Boolean algebras. Classes of algebras in which every congruence is uniquely determined by its classes are known as regular and classes of algebras in which every congruence is uniquely determined by its class containing a fixed constant are known as pointed regular (and 0-regular when the constant is denoted 0). Regular and 0-regular classes of algebras have received a good deal of attention in universal algebra. A Mal'cev condition for regular varieties were given in [4] and two distinct Mal'cev conditions for 0-regular varieties were given in [1:3, 14].

The ideals in universal algebra introduced in the case of multi operator groups by P.J. Higgins [20] and in the general case by R. Magari [27] and systematically studied in [41] and subsequent papers [17, 42, 43]. A. Ursini proposed a notion of ideal in 0-classes of algebras in [47]. His definition is a syntactic notion that abstracts the familiar closure properties of normal subgroups, ring ideals, etc. Although the 0-classes of congruences are ideals, in general not every ideal is the 0-class of a congruence, even in 0-regular varieties. In fact the 0-regular varieties in which the ideals are precisely the 0-classes of congruences are just the subtractive ones [17], i.e., those satisfying $\alpha(t, t) \approx 0$ and $\alpha(t, 0) \approx t$ for some binary term $\alpha$. More generally, an elegant theory of ideals in subtractive varieties has been worked out in [ [, [2] and [43]. Our notation is, more or less, standard; for general background in Universal Algebra we refer to [6, [1.9].

This paper is organized in four sections. In Section $\nabla$, we recall some definitions, properties and results relative to universal algebra, ideal determined varieties and rough set theory which will be used in the following sections of the paper. In Section B, we introduce the notions of rough approximation sets based on universal algebras and we study their properties. In section 四, we introduce the notion of rough subalgebras and rough ideals of an algebraic structure in an ideal determined variety as a generalization of the notion of subalgebras and ideals.

\section{A BRIEF EXCURSION INTO IDEAL DETERMINED VARIETIES AND ROUGH SET THEORY}

In this section we recall some definitions, properties and results relative to universal algebra, ideal determined varieties and rough set theory which will be used in the following sections of the paper.

2.1. universal algebra and ideal determined varieties. In this section, we will recall some definitions and results about ideals in universal algebras. A signature or language type is a set $\Sigma$ together with a mapping $\rho: \Sigma \longrightarrow w$. The elements of $\Sigma$ are called operation symbols. For each $\sigma \in \Sigma, \rho(\sigma)$ is called the rank of $\sigma$. In the sequel, for each $n \in w$, let $\Sigma_{n}=\{\sigma \mid \rho(\sigma)=n\}$. 
Let $\Sigma$ be a signature. A $\Sigma$-algebraic structure (structure, for short) is an ordered couple $\mathfrak{A}=\left(A,\left(\sigma^{\mathfrak{A}}: \sigma \in \Sigma\right)\right)$, where $A$ is a nonempty set and $\sigma^{\mathfrak{A}}$ is a map from $A^{\rho(\sigma)}$ to $A$, for all $\sigma \in \Sigma$. The set $A$ is called the universe of $\mathfrak{A}$ and the $\sigma^{\mathfrak{A}}$, s are called the fundamental operations of $\mathfrak{A}$. In the following, we prefer to write just $\sigma$ for $\sigma^{\mathfrak{A}}$ if this convention creates an ambiguity which seldom causes a problem.

In this paper, we shall use the following abbreviated notations: the sequences $x_{i}, \cdots, x_{j}$ and $\left(x_{i}, y_{i}\right), \cdots,\left(x_{j}, y_{j}\right)$ will be denoted by $x_{i}^{j}$ and $(x, y)_{i}^{j}$, respectively. For $j<i$ is the empty symbol. In this convention

$$
\sigma^{\mathfrak{A}}\left(x_{1}, \cdots, x_{i}, y_{i+1}, \cdots, y_{j}, z_{j+1}, \cdots, z_{\rho(\sigma)}\right)
$$

will be written as $\sigma^{\mathfrak{A}}\left(x_{1}^{i}, y_{i+1}^{j}, z_{j+1}^{\rho(\sigma)}\right)$.

Let $\mathfrak{A}$ be a $\Sigma$-algebraic structure and $B \subseteq A$. Then $B$ is called a subuniverse of $\mathfrak{A}$, if for all $\sigma \in \Sigma$ and $b_{1}^{\rho(\sigma)} \in B$ we have $\sigma^{\mathfrak{A}}\left(b_{1}^{\rho(\sigma)}\right) \in B$. The set of all subuniverses of $\mathfrak{A}$ will be denoted by $S u b(\mathfrak{A})$. It is well known that $(A, S u b(\mathfrak{A}))$ is an algebraic closed set system. In the sequel, The closure operator associated with the closed set system $(A, S u b(\mathfrak{A}))$ is denoted by $S g^{\mathfrak{A}}$.

Let $\mathfrak{A}$ be a $\Sigma$-algebraic structure. A binary relation $\Re$ on $A$ is called a congruence if it has the substitution property with respect to each $\sigma \in \Sigma$. The set of all congruences of $\mathfrak{A}$ will be denoted by $\operatorname{Con}(\mathfrak{A})$. It is well known that $\left(A^{2}, \operatorname{Con}(\mathfrak{A})\right)$ is an algebraic closed set system. In the sequel, The closure operator associated with the closed set system $\left(A^{2}, \operatorname{Con}(\mathfrak{A})\right)$ is denoted by $\operatorname{Con}^{\mathfrak{A}}$.

We will fix once and for all a class $\mathcal{K}$ of algebras of a fixed type, $\Sigma$, and assume that there is a distinguished nullary operation or else a constant, equationally definable in all algebras of $\mathcal{K}$, which we denote by 0 .

Definition 2.1. [41]

(1) A $\Sigma$-term $\alpha\left(t_{1}^{n}, s_{1}^{m}\right)$ in $\mathcal{K}$ is said to be a $\mathcal{K}$-ideal term in $s_{1}^{m}$ if $\alpha\left(t_{1}^{n}, \stackrel{m}{0}\right) \approx 0$ is an identity in $\mathcal{K}$. Obviously, 0 is a $\mathcal{K}$-ideal term.

(2) A non empty subset $I$ of $\mathfrak{A}$ in $\mathcal{K}$ is said to be a $\mathcal{K}$-ideal of $\mathfrak{A}$ if for each $\mathcal{K}$-ideal term $\alpha\left(t_{1}^{n}, s_{1}^{m}\right)$ in $s_{1}^{m}, a_{1}^{n} \in A$ and $i_{1}^{m} \in I$ we have $\alpha\left(a_{1}^{n}, i_{1}^{m}\right) \in I$. The set of all $\mathcal{K}$-ideals of $\mathfrak{A}$ is denoted by $I d_{\mathcal{K}}(\mathfrak{A})$. When there is no ambiguity we will drop the script $\mathcal{K}$. It is obvious that $\mathbf{0}=\{0\}, A \in \operatorname{Id}(\mathfrak{A})$.

Proposition 2.2. Let $\mathfrak{A}$ be an algebraic structure in $\mathcal{K}$. Then $(A ; \operatorname{Id}(\mathfrak{A}))$ is an algebraic closed set system.

Proof. Let $\mathcal{I}$ be a non empty family of ideals of $\mathfrak{A}$ and $\alpha\left(t_{1}^{n}, s_{1}^{m}\right)$ be an ideal term in $s_{1}^{m}$. Consider $a_{1}^{n} \in A$ and $i_{1}^{m} \in \cap \mathcal{I}$. Then for each $I \in \mathcal{I}$ we have $\alpha^{\mathfrak{A}}\left(a_{1}^{n}, i_{1}^{m}\right) \in I$ and it shows that $\alpha^{\mathfrak{A}}\left(a_{1}^{n}, i_{1}^{m}\right) \in \cap \mathcal{I}$. Hence, $(\mathfrak{A} ; \operatorname{Id}(\mathfrak{A}))$ is a closed set system. 
Now, assume that $\mathcal{I}$ is an upward directed non empty family of ideals of $\mathfrak{A}$ and $\alpha\left(t_{1}^{n}, s_{1}^{m}\right)$ be an ideal term in $s_{1}^{m}$. Consider $a_{1}^{n} \in A$ and $i_{1}^{m} \in \cup \mathcal{I}$. Hence, there is $I \in \mathcal{I}$ such that $a_{1}^{m} \in I$ and it implies that $\alpha^{\mathfrak{A}}\left(a_{1}^{n}, i_{1}^{m}\right) \in I \subseteq \cup \mathcal{I}$. Therefore, $(\mathfrak{A} ; \operatorname{Id}(\mathfrak{A}))$ is an algebraic closed set system.

By Proposition $[2.2$ we obtain that $\operatorname{Id}(\mathfrak{A})=(\operatorname{Id}(\mathfrak{A}) ; \subseteq)$ is an algebraic lattice. Let $\mathfrak{A}$ be an algebraic structure in the class $\mathcal{K}$. Let $I d_{\mathfrak{A}}^{\mathcal{K}}: \mathcal{P}(\mathfrak{A}) \longrightarrow \operatorname{Id}(\mathfrak{A})$ be the closure operator associated with the closed set system $(A ; \operatorname{Id}(\mathfrak{A}))$. When there is no ambiguity we will drop the superscript $\mathcal{K}$ or the subscript $\mathfrak{A}$. For a given subset $X$ of $A, \operatorname{Id}(X)$ are called the $\mathcal{K}$-ideal generated by $X$ in $\mathfrak{A}$.

Theorem 2.3. [I] Let $\mathfrak{A}$ be an algebraic structure in $\mathcal{K}$. Then for each subset $X$ of $A$ we have $I d(X)=\left\{\alpha^{\mathfrak{A}}\left(a_{1}^{n}, x_{1}^{m}\right) \mid \alpha\left(t_{1}^{n}, s_{1}^{m}\right)\right.$ is an ideal term in $\left.s_{1}^{m}, a_{1}^{n} \in A, x_{1}^{m} \in X\right\}$.

Lemma 2.4. Let $\mathfrak{A}$ be an algebraic structure in $\mathcal{K}, I$ be an ideal and $S$ be an subalgebra of $\mathfrak{A}$. If $S \in \mathcal{K}$ then $I \cap S$ is an ideal of the algebraic structure $S$.

Proof. It is straightforward.

Let $\mathfrak{A}$ and $\mathfrak{B}$ be algebraic structures in 0-class $\mathcal{K}$ and $h: \mathfrak{A} \longrightarrow \mathfrak{B}$ be a homomorphism. The preimage of 0 is called the kernel of $h$ and denoted by $\operatorname{ker}(h)$.

Proposition 2.5. Let $\mathfrak{A}$ and $\mathfrak{B}$ be algebraic structures in $\mathcal{K}$ and $h: \mathfrak{A} \longrightarrow \mathfrak{B}$ be a homomorphism.

(1) If $h$ is surjective and $I \in I d(\mathfrak{A})$ then $h(I) \in I d(\mathfrak{B})$.

(2) If $I \in I d(\mathfrak{B})$ then $h^{\leftarrow}(I) \in I d(\mathfrak{A})$ and $\operatorname{ker}(h) \subseteq h^{\leftarrow}(I)$. In particular, $\operatorname{ker}(h) \in \operatorname{Id}(\mathfrak{A})$.

(3) If $h$ is surjective and $X$ be a nonempty subset of $A$ then we have $h(\operatorname{Id}(X))=\operatorname{Id}(h(X))$.

Proof.

(1): Let $\alpha\left(t_{1}^{n}, s_{1}^{m}\right)$ be an ideal term, $b_{1}^{n} \in B$ and $j_{i}^{m} \in h(I)$. Thus there are $a_{1}^{n} \in A$ and $i_{1}^{m} \in I$ such that $h\left(a_{d}\right)=b_{d}$ for each $1 \leq d \leq n$ and $h\left(i_{d}\right)=j_{d}$ for each $1 \leq d \leq m$. Therefore,

$$
\begin{aligned}
\alpha\left(b_{1}^{n}, j_{1}^{m}\right) & =\alpha\left(h\left(a_{1}\right), \cdots, h\left(a_{n}\right), h\left(i_{1}\right), \cdots, h\left(i_{m}\right)\right) \\
& =h\left(\alpha\left(a_{1}^{n}, i_{1}^{m}\right)\right) \in h(I) .
\end{aligned}
$$

It shows that $h(I)$ is an ideal of $\mathfrak{B}$.

(2): Let $\alpha\left(t_{1}^{n}, s_{1}^{m}\right)$ be an ideal term, $a_{1}^{n} \in B$ and $j_{i}^{m} \in h^{\leftarrow}(I)$. Then we have

$$
h\left(\alpha\left(a_{1}^{n}, j_{1}^{m}\right)\right)=\alpha\left(h\left(a_{1}\right), \cdots, h\left(a_{n}\right), h\left(j_{1}\right), \cdots, h\left(j_{m}\right)\right) \in I .
$$

So $\alpha\left(a_{1}^{n}, j_{1}^{m}\right) \in h^{\leftarrow}(I)$ and it shows that $h^{\leftarrow}(I)$ is an ideal of $\mathfrak{A}$. Also, we have $0 \in I$ and it implies that $h^{\leftarrow}(0) \subseteq h^{\leftarrow}(I)$. 
(3): By Theorem [2.3], one can see that $h(\operatorname{Id}(X)) \subseteq \operatorname{Id}(h(X))$. Now, let $b \in \operatorname{Id}(h(X))$. Hence, there is an ideal term $\alpha\left(t_{1}^{n}, s_{1}^{m}\right)$ and $b_{1}^{n} \in B$ and $j_{i}^{m} \in h(X)$ such that $b=\alpha\left(b_{1}^{n}, j_{1}^{m}\right)$. It implies that there are $a_{1}^{n} \in A$ and $x_{1}^{m} \in X$ such that $h\left(a_{d}\right)=b_{d}$ for each $1 \leq d \leq n$ and $h\left(x_{d}\right)=j_{d}$ for each $1 \leq d \leq m$. Therefore,

$$
\begin{aligned}
b=\alpha\left(b_{1}^{n}, j_{1}^{m}\right) & =\alpha\left(h\left(a_{1}\right), \cdots, h\left(a_{n}\right), h\left(x_{1}\right), \cdots, h\left(x_{m}\right)\right) \\
& =h\left(\alpha\left(a_{1}^{n}, x_{1}^{m}\right)\right) \in h(\operatorname{Id}(X)) .
\end{aligned}
$$

It shows that $h(\operatorname{Id}(X))=\operatorname{Id}(h(X))$.

Corollary 2.6. Let $\mathfrak{A}$ and $\mathfrak{B}$ be algebraic structures in $\mathcal{K}$ and $h: \mathfrak{A} \longrightarrow \mathfrak{B}$ be an epimorphism. Then the mapping

$$
\begin{aligned}
h_{I d}: \mathbf{I d}(\mathfrak{A}) & \longrightarrow \mathbf{I d}(\mathfrak{B}) \\
I & \longmapsto h(I)
\end{aligned}
$$

is a complete join semilattice epimorphism.

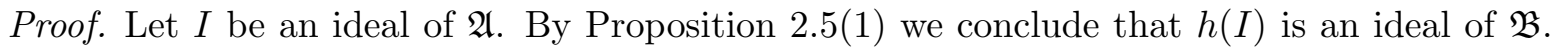
Assume $J$ be an ideal of $\mathfrak{B}$. Thus by Proposition $\left[2.5(\mathbb{Z})\right.$ we have $h^{\leftarrow}(J) \in \operatorname{Id}(\mathfrak{A})$ and since $h$ is surjective then $h\left(h^{\leftarrow}(J)\right)=J$. It shows that $h_{I d}$ is a surjection. Now, let $\mathcal{I} \subseteq \operatorname{Id}(\mathfrak{A})$. By Proposition [2.5(B) we have

$$
h(\vee \mathcal{I})=h(I d(\cup \mathcal{I}))=I d(h(\cup \mathcal{I}))=\cup_{I \in \mathcal{I}} I d(h(I))=\vee_{I \in \mathcal{I}} h(I) .
$$

It shows that $h_{I d}$ is a complete join semilattice epimorphism.

Definition 2.7. A 0 -class $\mathcal{K}$ is called ideal determined if for each $\mathfrak{A} \in \mathcal{K}$ there is an isomorphism, ${ }^{\delta_{\mathfrak{A}}}: \operatorname{Id}(\mathfrak{A}) \longrightarrow \operatorname{Con}(\mathfrak{A})$, between the lattice of congruences of $\mathfrak{A}$ and the lattice of ideals of $\mathfrak{A}$. It means that each ideal $I$ of $\mathfrak{A}$ is a 0 -class of a unique congruence relation $I^{\delta_{\mathfrak{A}}}$. It is clear that $\mathbf{0}^{\delta_{\mathfrak{A}}}=\Delta_{\mathfrak{A}}$ and $A^{\delta_{\mathfrak{A}}}=\nabla_{\mathfrak{A}}$. When there is no ambiguity we will drop the script $\mathfrak{A}$.

It is clear that a 0 -class $\mathcal{K}$ is ideal determined if congruences of $\mathfrak{A} \in \mathcal{K}$ are uniquely determined by their 0 -classes. A 0 -class $\mathcal{K}$ is called 0 -regular if congruences of algebras in $\mathcal{K}$ are uniquely determined by their 0-classes.

Proposition 2.8. [14] Let $\mathcal{V}$ is a 0-variety. Then $\mathcal{V}$ is 0 -regular if and only if there are an integer $n$, binary terms $d_{1}, \cdots, d_{n}$ and quaternary terms $q_{1}, \cdots, q_{n}$ such that the following 
identities hold.

$$
\begin{array}{rlrl}
d_{i}(x, x) & \approx 0 & & \text { for } 1 \leq i \leq n \\
x & \approx q_{1}\left(x, y, 0, d_{1}(x, y)\right) & \\
q_{i}\left(x, y, d_{i}(x, y), 0\right) & \approx q_{i+1}\left(x, y, 0, d_{i+1}(x, y)\right) & & \text { for } 1 \leq i \leq n \\
q_{n}\left(x, y, d_{n}(x, y), 0\right) & \approx y & &
\end{array}
$$

In the following we assume that $\mathcal{V}$ is a pointed variety which is ideal determined.

Lemma 2.9. [17] Let $\mathfrak{A}$ be an algebraic structure in $\mathcal{V}$ and $I$ be an ideal of $\mathfrak{A}$. Then

$$
\left(a_{1}, a_{2}\right) \in I^{\delta} \text { if and only if } d_{i}\left(a_{1}, a_{2}\right) \in I \text {, for each } 1 \leq i \leq n,
$$

where $d_{1}, \cdots, d_{n}$ are terms from Proposition [2.8.

Proposition 2.10. Let $\mathfrak{A}$ and $\mathfrak{B}$ be algebraic structures in $\mathcal{V}$ and $h: \mathfrak{A} \longrightarrow \mathfrak{B}$ be a homomorphism. Then for each $I \in I d(\mathfrak{B})$ we have $h^{\leftarrow}\left(I^{\delta_{\mathfrak{B}}}\right)=\left(h^{\leftarrow}(I)\right)^{\delta_{\mathfrak{A}}}$. In particular, $\operatorname{ker}^{\delta_{\mathfrak{A}}}(h)=\kappa(h)$.

Proof. Let $\left(a_{1}, a_{2}\right) \in h^{\leftarrow}\left(I^{\delta_{\mathfrak{B}}}\right)$. Therefore, $\left(h\left(a_{1}\right), h\left(a_{2}\right)\right) \in I^{\delta_{\mathfrak{B}}}$ and by Lemma [2耳 we get that $d_{i}\left(h\left(a_{1}\right), h\left(a_{2}\right)\right) \in I$, for each $1 \leq i \leq n$. It implies that $h\left(d_{i}\left(a_{1}, a_{2}\right)\right) \in I$, for each $1 \leq i \leq n$, and consequently we have $d_{i}\left(a_{1}, a_{2}\right) \in h^{\leftarrow}(I)$, for each $1 \leq i \leq n$. On the other

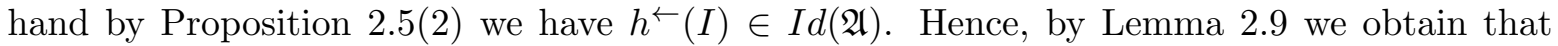
$\left(a_{1}, a_{2}\right) \in\left(h^{\leftarrow}(I)\right)^{\delta_{\mathfrak{A}}}$.

Conversely, by Proposition [2.5(四) we know that $h^{\leftarrow}(I)$ is an ideal of $\mathfrak{A}$ so the congruence relation $\left(h^{\leftarrow}(I)\right)^{\delta_{\mathfrak{A}}}$ exists. Now, assume that $\left(a_{1}, a_{2}\right) \in\left(h^{\leftarrow}(I)\right)^{\delta_{\mathfrak{A}}}$. By Lemma [2.9] we conclude that $d_{i}\left(a_{1}, a_{2}\right) \in h^{\leftarrow}(I)$, for each $1 \leq i \leq n$. It implies that $h\left(d_{i}\left(a_{1}, a_{2}\right)\right) \in I$. So $d_{i}\left(h\left(a_{1}\right), h\left(a_{2}\right)\right) \in I$, for each $1 \leq i \leq n$ and it means that $\left(h\left(a_{1}\right), h\left(a_{2}\right)\right) \in I^{\delta_{\mathfrak{A}}}$. Thus $\left(a_{1}, a_{2}\right) \in h^{\leftarrow}\left(I^{\delta_{\mathfrak{B}}}\right)$.

In particular, we have $\operatorname{ker}^{\delta_{\mathfrak{A}}}(h)=\left(h^{\leftarrow}(0)\right)^{\delta_{\mathfrak{A}}}=h^{\leftarrow}\left(0^{\delta_{\mathfrak{B}}}\right)=h^{\leftarrow}\left(\Delta_{\mathfrak{B}}\right)=\kappa(h)$.

Corollary 2.11. Let $\mathfrak{A}$ and $\mathfrak{B}$ be algebraic structures in $\mathcal{V}$ and $h: \mathfrak{A} \longrightarrow \mathfrak{B}$ be a homomorphism. Then $h$ is injective if and only if $\operatorname{ker}(h)=0$.

Proof. If $h$ is injective then by Proposition ए.]1, $\operatorname{ker}^{\delta_{\mathfrak{A}}}(h)=\Delta_{\mathfrak{A}}$ and it implies that $\operatorname{ker}(h)=0$. Conversely, if $\operatorname{ker}(h)=0$ then $\kappa(h)=\Delta_{\mathfrak{A}}$ and it shows that $h$ is injective.

Let $\mathfrak{A}=(A ; \leq)$ be a poset. In the following, the interval $\left\{a \in A \mid a_{1} \leq a \leq a_{2}\right\}$ is denoted by $\mathfrak{A}\left[a_{1}, a_{2}\right]$. 
Corollary 2.12. (Ideal Correspondence Theorem) Let $\mathfrak{A}$ and $\mathfrak{B}$ be algebraic structures in $\mathcal{V}$ and $h: \mathfrak{A} \longrightarrow \mathfrak{B}$ be an epimorphism. Then the mapping

$$
\begin{aligned}
h_{I d}: \operatorname{Id}(\mathfrak{A})[\operatorname{ker}(h), A] & \longrightarrow \operatorname{Id}(\mathfrak{B}) \\
I & \longmapsto h(I)
\end{aligned}
$$

is a complete lattice isomorphism. (see Figure प)

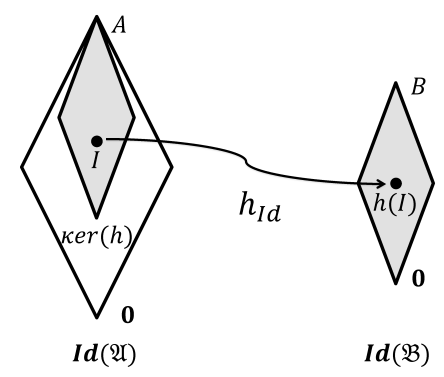

Figure 1. Ideal Correspondence Theorem

Proof. By Corollary [2.6, $h_{I d}$ is a complete join semilattice epimorphism. Now, let $I_{1}$ and $I_{2}$ be ideals of $\mathfrak{A}$ containing $\operatorname{ker}(h)$ such that $h_{I d}\left(I_{1}\right)=h_{I d}\left(I_{2}\right)$. Assume that $a_{1} \in h\left(I_{1}\right)$. So there is $a_{2} \in I_{2}$ such that $h\left(a_{1}\right)=h\left(a_{2}\right)$ and this states that $\left(a_{1}, a_{2}\right) \in \kappa(h)$. By Proposition [2.10, we have $\kappa(h)=\operatorname{ker}^{\delta_{\mathfrak{A}}}(h)$ and it implies that $\left(a_{1}, a_{2}\right) \in I_{2}^{\delta_{\mathfrak{A}}}$. On the other hand, we have $\left(a_{2}, 0\right) \in I_{2}^{\delta_{\mathfrak{A}}}$ and it implies that $\left(a_{1}, 0\right) \in I_{2}^{\delta_{\mathfrak{A}}}$. Hence, $a_{1} \in 0 / I_{2}^{\delta_{\mathfrak{A}}}=I_{2}$ and it shows $I_{1} \subseteq I_{2}$. Similarly, $I_{2} \subseteq I_{1}$ and it means that $h_{I d}$ is an injection.

Now, let $\mathcal{I} \subseteq \operatorname{Id}(\mathfrak{A})[\operatorname{ker}(h), A]$. It is well known that $h_{I d}(\cap \mathcal{I}) \subseteq \cap_{I \in \mathcal{I}} h(I)$. Let $b \in \cap_{I \in \mathcal{I}} h(I)$. Hence, there are $a_{I} \in I$, for each $I \in \mathcal{I}$ such that $b=h\left(a_{I}\right)$. Fix $J \in \mathcal{I}$ and consider $a_{J}$. For each $I \in \mathcal{I}$ we have $\left(a_{I}, a_{J}\right) \in \kappa(h)=\operatorname{ker}^{\delta_{\mathfrak{A}}}(h) \subseteq I^{\delta_{\mathfrak{A}}}$ and it states that $a_{J} \in I$. Thus $b=h\left(a_{J}\right) \in \cap \mathcal{I}$ and it means $\cap_{I \in \mathcal{I}} h(I) \subseteq h_{I d}(\cap \mathcal{I})$. Therefore, $h_{I d}$ is a complete lattice isomorphism.

Theorem 2.13. (Congruence Correspondence Theorem) Let $\mathfrak{A}$ and $\mathfrak{B}$ be two $\Sigma$-algebraic structures and $h: \mathfrak{A} \longrightarrow \mathfrak{B}$ be an epimorphism. Then the mapping

$$
\begin{aligned}
h_{C o n}: \operatorname{Con}(\mathfrak{A})\left[\kappa(h), \nabla_{\mathfrak{A}}\right] & \longrightarrow \operatorname{Con}(\mathfrak{B}) \\
\Re & \longmapsto
\end{aligned}
$$

is a complete lattice isomorphism. (see Figure 回)

Proof. It is straightforward. 


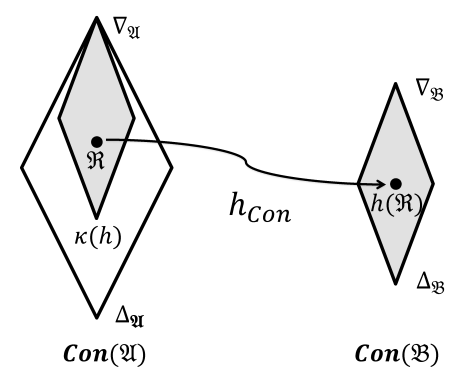

Figure 2. Congruence Correspondence Theorem

Theorem 2.14. Let $\mathfrak{A}$ and $\mathfrak{B}$ be algebraic structures in $\mathcal{V}$ and $h: \mathfrak{A} \longrightarrow \mathfrak{B}$ be an epimorphism. Then for each ideal I of $\mathfrak{A}$ containing $\operatorname{ker}(h)$ we have $h_{C o n}\left(I^{\delta_{\mathfrak{A}}}\right)=h_{I d}^{\delta_{\mathfrak{B}}}(I)$. i. e. the following diagram is commutative.



FIGURE 3

Proof. Let $I$ be an ideal of $\mathfrak{A}$ containing $\operatorname{ker}(h)$ and consider $\left(b_{1}, b_{2}\right) \in h_{I d}^{\delta_{\mathfrak{B}}}(I)$. So there are $a_{1}, a_{2} \in A$ such that $b_{1}=h\left(a_{1}\right)$ and $b_{2}=h\left(a_{2}\right)$. By Lemma [2.9 we have $h\left(d_{i}\left(a_{1}, a_{2}\right)\right) \in h_{I d}(I)$, for each $1 \leq i \leq n$. Hence, for each $1 \leq i \leq n$ there are $a_{i} \in I$ such that $h\left(d_{i}\left(a_{1}, a_{2}\right)\right)=h_{I d}\left(a_{i}\right)$. By Proposition [2.] we have $\left(a_{i}, d_{i}\left(a_{1}, a_{2}\right)\right) \in \kappa(h)=\operatorname{ker}^{\delta_{\mathfrak{A}}}(h)$ and by hypothesis we get that $\left(a_{i}, d_{i}\left(a_{1}, a_{2}\right)\right) \in I^{\delta_{\mathfrak{A}}}$, for each $1 \leq i \leq n$. Consequently, $d_{i}\left(a_{1}, a_{2}\right) \in I$ for each $1 \leq i \leq n$ and it shows that $\left(a_{1}, a_{2}\right) \in I^{\delta_{\mathfrak{A}}}$. Hence, $\left(b_{1}, b_{2}\right)=\left(h\left(a_{1}\right), h\left(a_{2}\right)\right) \in h\left(I^{\delta_{\mathfrak{A}}}\right)$ and it states that $h_{\text {Id }}^{\delta_{\mathfrak{B}}}(I) \subseteq h_{\text {Con }}\left(I^{\delta_{\mathfrak{A}}}\right)$.

Conversely, let $I$ be an ideal of $\mathfrak{A}$ containing $\operatorname{ker}(h)$. By Proposition $2 . \mathbb{J}$ we have $\kappa(h)=$ $\operatorname{ker}^{\delta_{\mathfrak{A}}}(h) \subseteq I^{\delta_{\mathfrak{A}}}$ and it means that $h_{C o n}\left(I^{\delta_{\mathfrak{A}}}\right)$ is well defined. Assume that $\left(b_{1}, b_{2}\right) \in h_{C o n}\left(I^{\delta_{\mathfrak{A}}}\right)$. Thus there is $\left(a_{1}, a_{2}\right) \in I^{\delta_{\mathfrak{A}}}$ such that $\left(b_{1}, b_{2}\right)=\left(h\left(a_{1}\right), h\left(a_{2}\right)\right)$. It concludes that $d_{i}\left(b_{1}, b_{2}\right)=$ $d_{i}\left(h\left(a_{1}\right), h\left(a_{2}\right)\right)=h\left(d_{i}\left(a_{1}, a_{2}\right)\right) \in h(I)=h_{I d}(I)$, for each $1 \leq i \leq n$. So $\left(b_{1}, b_{2}\right) \in h_{I d}^{\delta_{\mathfrak{B}}}(I)$ and it means that $h_{C o n}\left(I^{\delta_{\mathfrak{A}}}\right) \subseteq h_{I d}^{\delta_{\mathfrak{B}}}(I)$. 
Let $\mathcal{K}$ be an ideal determined class of algebras, $\mathfrak{A} \in \mathcal{K}$ and $I$ be an ideal of $\mathfrak{A}$. For each $a \in A$, the equivalence class of $a / I^{\delta}$ is denoted by $a / I$ and the set of all equivalence classes $A / I^{\delta}$ is denoted by $A / I$. Also, the quotient algebra $\mathfrak{A} / I^{\delta}$ is denoted by $\mathfrak{A} / I$.

Lemma 2.15. Let $\mathfrak{A}$ and $\mathfrak{B}$ be two algebraic structures in $\mathcal{V}$. If $h: \mathfrak{A} \longrightarrow \mathfrak{B}$ is an epimorphism and $I$ is an ideal of $\mathfrak{A}$ containing $\operatorname{ker}(h)$ then for any $a \in A$ we have $h(a / I)=h(a) / h(I)$.

Proof. Let $b \in h(a / I)$. So there is $a^{\prime} \in a / I$ such that $b=h\left(a^{\prime}\right)$. Therefore, $\left(a, a^{\prime}\right) \in I^{\delta_{\mathfrak{A}}}$ and it implies that $(h(a), b) \in h\left(I^{\delta_{\mathfrak{A}}}\right)$. Since, $I$ contains $\operatorname{ker}(h)$ so by Theorem [.14, we have $h_{C o n}\left(I^{\delta_{\mathfrak{A}}}\right)=h_{I d}^{\delta_{\mathfrak{B}}}(I)$ and it implies that $(h(a), b) \in h_{I d}^{\delta_{\mathfrak{B}}}(I)$. Thus $b \in h(a) / h(I)$.

Now, let $b \in h(a) / h(I)$. So $(b, h(a)) \in h_{I d}^{\delta_{\mathfrak{B}}}(I)$ and by Theorem [2.14 we conclude that $(b, h(a)) \in h_{C o n}\left(I^{\delta_{\mathfrak{A}}}\right)$. Therefore, exists $\left(a_{1}, a_{2}\right) \in I^{\delta_{\mathfrak{A}}}$ such that $(b, h(a))=\left(h\left(a_{1}\right), h\left(a_{2}\right)\right)$. This states that $b=h\left(a_{1}\right)$ and $\left(a, a_{2}\right) \in \kappa(h)=\operatorname{ker}^{\delta_{\mathfrak{A}}}(h) \subseteq I^{\delta_{\mathfrak{A}}}$. So $\left(a_{1}, a\right) \in I^{\delta_{\mathfrak{A}}}$ and this means $a_{1} \in a / I$ and sequently we obtain $b \in h(a / I)$. It shows that $h(a) / h(I) \subseteq h(a / I)$.

Lemma 2.16. Let $\mathfrak{A}$ and $\mathfrak{B}$ be two algebraic structures in $\mathcal{V}$. If $h: \mathfrak{A} \longrightarrow \mathfrak{B}$ is a homomorphism and $I$ is an ideal of $\mathfrak{B}$ then for each $a \in A$ we have $a / h^{\leftarrow}(I)=h^{\leftarrow}(h(a) / I)$. Moreover, $h\left(a / h^{\leftarrow}(I)\right) \subseteq h(a) / I$.

Proof. According to Proposition [2.5(区) we know that $h^{\leftarrow}(I)$ is an ideal of $\mathfrak{A}$. According to Proposition [2.10, we have

$$
\begin{aligned}
x \in a / h^{\leftarrow}(I) & \Longleftrightarrow(x, a) \in\left(h^{\leftarrow}(I)\right)^{\delta_{\mathfrak{A}}}=h^{\leftarrow}\left(I^{\delta_{\mathfrak{B}}}\right) \\
& \Longleftrightarrow(h(x), h(a)) \in I^{\delta_{\mathfrak{B}}} \\
& \Longleftrightarrow h(x) \in h(a) / I \\
& \Longleftrightarrow x \in h^{\leftarrow}(h(a) / I) .
\end{aligned}
$$

It shows that the equality holds. Also, we can conclude that $h\left(a / h^{\leftarrow}(I)\right)=h\left(h^{\leftarrow}(h(a) / I)\right) \subseteq$ $h(a) / I$, for each $a \in A$.

2.2. Rough set theory. We recall in this section some basic facts about the Galois connections and the rough set theory based on formal context.

Definition 2.17. Let $\mathscr{A}=(A ; \leq)$ and $\mathscr{B}=(B ; \preccurlyeq)$ be posets and $f: A \longrightarrow B$ be a map between posets.

(1) $f$ is monotone if $a_{1} \leq a_{2}$ implies $f\left(a_{1}\right) \preccurlyeq f\left(a_{2}\right)$, for all $a_{1}, a_{2} \in A$.

(2) $f$ is antitone if $a_{1} \leq a_{2}$ implies $f\left(a_{2}\right) \preccurlyeq f\left(a_{1}\right)$, for all $a_{1}, a_{2} \in A$.

In particular case which $\mathscr{A}=\mathscr{B}$,

(1) $f$ is inflationary (also called extensive) if $a \leq f(a)$ for all $a \in A$. 
(2) $f$ is deflationary if $f(a) \leq a$ for all $a \in A$.

(3) $f$ is idempotent if $f^{2}=f$.

(4) $f$ is a closure operator on $\mathscr{A}$ if it is inflationary, monotone and idempotent.

(5) $f$ is a kernel operator (also called interior operator) on $\mathscr{A}$ if it is deflationary, monotone and idempotent.

A fixpoint of a closure operator or a kernel operator $f$, i.e. an element $a$ of $A$ that satisfies $f(a)=a$, is called a closed element of $f$. The set of closed elements of $f$ will be denoted by $\mathscr{C}_{f}$.

Definition 2.18. Let $\mathscr{A}=(A ; \leq)$ and $\mathscr{B}=(B ; \preccurlyeq)$ be posets. Suppose that $f: A \longrightarrow B$ and $g: B \longrightarrow A$ are functions. The pair $(f, g)$ is called a covariant (or monotone) Galois connection between $\mathscr{A}$ and $\mathscr{B}$ if for all $a \in A$ and $b \in B$ we have

$$
a \leq g(b) \text { if and only if } f(a) \preccurlyeq b .
$$

Proposition 2.19. [18] Let $\mathscr{A}$ and $\mathscr{B}$ be posets and $f: A \longrightarrow B$ and $g: B \longrightarrow A$ be two functions. Then the following assertions are equivalent:

(1) $(f, g)$ is a covariant Galois connection between $\mathscr{A}$ and $\mathscr{B}$.

(2) $f$ and $g$ are monotone functions, $g f$ is inflationary and $f g$ is deflationary functions.

Proposition 2.20. [110] Let $\mathscr{A}$ and $\mathscr{B}$ be posets and $(f, g)$ is a covariant Galois connection between $\mathscr{A}$ and $\mathscr{B}$. Then the following assertions hold:

(1) $f g f=f$ and $g f g=g$.

(2) If $\vee X$ exists for some $X \subseteq A$ then $\vee f(X)$ exists and $\vee f(X)=f(\vee X)$.

(3) If $\wedge Y$ exists for some $Y \subseteq B$ then $\wedge g(Y)$ exists and $\wedge g(Y)=g(\wedge Y)$.

(4) $f(a)=\min \{b \in B \mid a \leq g(b)\}, g(b)=\max \{a \in A \mid f(a) \preccurlyeq b\}$.

(5) $g f$ is a closure operator on $\mathscr{A}$ and $\mathscr{C}_{g f}=g(B)$.

(6) $f g$ is a kernel operator on $\mathscr{B}$ and $\mathscr{C}_{f g}=f(A)$.

Definition 2.21. A formal context is a triple $(A, B$; $)$, where $A$ and $B$ are sets, $\Re \subseteq A \times B$ is a relation from $A$ to $B$. In a formal context $(A, B ; \Re), A$ is interpreted as the set of objects, $B$ the set of properties, and $(a, b) \in \Re$ reads as that the object $a$ has property $b$.

An object $a \in A$ has the set of properties:

$$
a \Re=\{b \in B \mid(a, b) \in \Re\} .
$$

A property $b \in B$ is possessed by the set of objects:

$$
\Re b=\{a \in A \mid(a, b) \in \Re\} .
$$

It is clear that if $A=B$ and $\Re$ is an equivalence relation on $A$ then we have $a \Re=\Re a=\Re[a]$. 
Definition 2.22. [16] Let $(A, B ; \Re)$ be a formal context. Define a pair of operators $\left(\Re_{\exists}, \Re^{\forall}\right)$ between the powersets of $A$ and $B$ as follows:

$$
\begin{aligned}
\Re_{\exists}: \mathcal{P}(A) \longrightarrow \mathcal{P}(B), \Re_{\exists}(X) & =\{b \in B \mid \exists x \in X,(x, b) \in \Re\} \\
& =\{b \in B \mid \Re b \cap X \neq \emptyset\} \\
& =\cup_{x \in X} x \Re . \\
\Re^{\forall}: \mathcal{P}(B) \longrightarrow \mathcal{P}(A), \Re^{\forall}(Y)=\{a & \in A \mid \forall b \in B, \quad((a, b) \in \Re \rightarrow b \in Y)\} \\
=\{a & \in A \mid a \Re \subseteq Y\} .
\end{aligned}
$$

Proposition 2.23. Let $(A, B ; \Re)$ be a formal context. Then the pair $\left(\Re_{\exists}, \Re^{\forall}\right)$ is a covariant Galois connection between the posets $\mathcal{P}(A)$ and $\mathcal{P}(B)$.

Proof. It is obvious that $X \subseteq \Re^{\forall}(Y)$ if and only if $\Re_{\exists}(X) \subseteq Y$ and by Definition [2.18 it implies that the pair $\left(\Re_{\exists}, \Re^{\forall}\right)$ is a covariant Galois connection between the posets $\mathcal{P}(A)$ and $\mathcal{P}(B)$.

Corollary 2.24. Let $(A, B ; \Re)$ be a formal context. Then the following assertions hold for any family $\{X\} \cup\left\{X_{i}\right\}_{i \in I} \subseteq \mathcal{P}(A)$ and $\{Y\} \cup\left\{Y_{i}\right\}_{i \in I} \subseteq \mathcal{P}(B)$ :

(1) $\Re_{\exists}$ and $\Re^{\forall}$ are monotone functions.

(2) $\Re_{\exists} \Re^{\forall} \Re_{\exists}=\Re_{\exists}$ and $\Re^{\forall} \Re_{\exists} \Re^{\forall}=\Re^{\forall}$.

(3) $\cup_{i \in I} \Re_{\exists}\left(X_{i}\right)=\Re_{\exists}\left(\cup_{i \in I} X_{i}\right)$.

(4) $\cap_{i \in I} \Re_{\forall}\left(Y_{i}\right)=\Re_{\forall}\left(\cap_{i \in I} Y_{i}\right)$.

(5) $\Re_{\exists}(X)=\cap\left\{Y \in \mathcal{P}(B) \mid X \subseteq \Re_{\forall}(Y)\right\}$.

(6) $\Re_{\forall}(Y)=\cup\left\{X \in \mathcal{P}(B) \mid \Re_{\exists}(X) \subseteq Y\right\}$.

(7) $\Re_{\forall} \Re_{\exists}$ is a closure operator on $\mathcal{P}(A)$ and $\mathscr{C}_{\Re_{\forall} \Re_{\exists}}=\Re_{\forall}(\mathcal{P}(B))$.

(8) $\Re_{\exists} \Re_{\forall}$ is a kernel operator on $\mathcal{P}(B)$ and $\mathscr{C}_{\Re_{\exists} \Re_{\forall}}=\Re_{\exists}(\mathcal{P}(A))$.

Proof. It is straightforward by Proposition [2.20] and [2.23].

Definition 2.25. [16] Let $(A, B ; \Re)$ be a formal context. A pair $(X, Y) \in \mathcal{P}(A) \times \mathcal{P}(B)$ is called a property oriented concept (or, concepts based on rough set theory) of a context $(A, B ; \Re)$ if $\Re_{\exists}(X)=Y$ and $\Re^{\forall}(Y)=X$. The set of all property oriented concepts of a context $(A, B ; \Re)$ is denoted by $\mathfrak{O C}(A, B ; \Re)$.

Proposition 2.26. Let $(A, B ; \Re)$ be a formal context. The following assertion holds:

$$
\mathfrak{O C}(A, B ; \Re)=\left\{\left(X, \Re_{\exists}(X)\right) \mid X \in \mathscr{C}_{\Re_{\exists} \Re^{\forall}}\right\}
$$


Alg. Struc. Appl. Vol. 6 No. 1 (2019) 1-21.

Proof. Let $(X, Y) \in \mathfrak{D C}(A, B ; \Re)$. So $\Re_{\exists}(X)=Y$ and $\Re^{\forall}(Y)=X$ and by Corollary [2.24(8) it implies that $X \in \mathscr{C}_{\Re \ni \Re^{\forall}}$ and $(X, Y)=\left(\Re^{\forall}(Y), \Re_{\exists}\left(\Re^{\forall}(Y)\right)\right)$. Conversely, it is obvious that $\left\{\left(X, \Re_{\exists}(X)\right) \mid X \in \mathscr{C}_{\Re_{\exists} \Re^{\forall}}\right\} \subseteq \mathfrak{O C C}(A, B ; \Re)$ and it shows the equality.

Proposition 2.27. Let $(A, A ; \Re)$ be a formal context. The following assertions hold:

(1) If $\Re a=a \Re$ for each $a \in A$, then $\Re^{\forall}(X) \subseteq \Re_{\exists}(X)$ for any $X \subseteq A$. In particular, if $\Re$ is a symmetric relation then $\Re^{\forall}(X) \subseteq \Re_{\exists}(X)$ for any $X \subseteq A$.

(2) If $\Re$ is a reflexive relation then $\Re^{\forall}(X) \subseteq X \subseteq \Re_{\exists}(X)$.

(3) If $\Re$ is an equivalence relation then $\Re^{\forall}(X)=\{a \in A \mid \Re[a] \subseteq X\}$ and $\Re_{\exists}(X)=\{a \in$ $A \mid \Re[a] \cap X \neq \emptyset\}$.

Proof.

(1) Let $\Re a=a \Re$ for each $a \in A$. Assume that $a \in \Re^{\forall}(X)$. It implies that $a \Re \subseteq X$ and it means that $\Re a \subseteq X$. So $a \in \Re_{\exists}(X)$.

[2] Let $\Re$ be a reflexive relation and $a \in \Re^{\forall}(X)$. So we have $(a, a) \in \Re$ and it implies that $a \in X$. If $x \in X$, then $x \in \Re x \cap X$ and it shoes that $x \in \Re_{\exists}(X)$.

(3) It is straightforward.

In the theory of rough sets, presented by Pawlak, equivalence relations are very important. Equivalence classes are basic building blocks for lower and upper approximations of a subset of the universe set.

Let $A$ be a non empty finite set called the universe set and let $\Re$ be an equivalence relation on $A$. Then, $(A, \Re)$ is called an approximation space. By a rough approximation in $(A, \Re)$ we mean a mapping $A p r_{\Re}^{A}: \mathcal{P}(A) \longrightarrow \mathcal{P}(A) \times \mathcal{P}(A)$ defined for every $X \in \mathcal{P}(A)$ by

$$
\operatorname{Apr}_{\Re}^{A}(X)=\left(\underline{A p r}_{\Re}^{A}(X), \overline{A p r}_{\Re}^{A}(X)\right),
$$

where $\underline{A p r}_{\Re}^{A}(X)=\Re^{\forall}(X)$ and $\overline{A p r}_{\Re}^{A}(X)=\Re^{\exists}(X)$. $\underline{A p r}_{\Re}^{A}(X)$ and $\overline{A p r}_{\Re}^{A}(X)$ are called the lower rough approximation and the upper rough approximation of $X$ in $(A, \Re)$, respectively. When there is no ambiguity we will drop the superscript $A$. A subset $X$ of $A$ is called definable with respect to $\Re$ if $\underline{A p r}_{\theta}(X)=\overline{A p r}_{\theta}(X)$.

\section{Rough approximation sets based on universal algebras}

Let $\mathcal{K}$ be an ideal determined class and $I$ be an ideal of $\mathfrak{A} \in \mathcal{K}$. In the following, the approximation space $\left(A ; I^{\delta}\right)$ is denoted by $(\mathfrak{A} ; I)$ and it is named the approximation space induced by $I$. Also, $\underline{A p r}_{I^{\delta}}^{A}(X)$ and $\overline{A p r}_{I^{\delta}}^{A}(X)$ are denoted by $\underline{A p r}_{I}^{\mathfrak{A}}(X)$ and $\overline{A p r}_{I}^{\mathfrak{A}}(X)$ and they are called the lower rough and the upper rough approximation on $\mathfrak{A}$ induced by $I$, respectively. When there is no ambiguity we will drop the superscript $\mathfrak{A}$. 
By a rough approximation in $(\mathfrak{A} ; I)$ we mean a mapping $A p r_{I}: \mathcal{P}(A) \longrightarrow \mathcal{P}(A) \times \mathcal{P}(A)$ defined for every $X \in \mathcal{P}(A)$ by

$$
\operatorname{Apr}_{I}(X)=\left(\underline{A p r}_{I}(X), \overline{A p r}_{I}(X)\right)
$$

A subset $X$ of $A$ is called definable with respect to $I$ if $\underline{A p r}_{I}(X)=\overline{A p r}_{I}(X)$.

Proposition 3.1. Let $(\mathfrak{A} ; I)$ be an approximation space. Then for any subsets $X$ and $Y$ of $A$ the following assertions hold:

(1) $\operatorname{Apr}_{I}(X) \subseteq X \subseteq \overline{\operatorname{Apr}}_{I}(X)$,

(2) $X$ is definable with respect to $I$ if and only if $X$ is the union of congruence classes of $I^{\delta}$. In particular, $A, \emptyset, \overline{A p r}_{I}(X), \underline{A p r}_{I}(X)$ and $x / I$ are definable sets with respect to $I$.

(3) $X$ is definable with respect to $I$ if and only if $\underline{A p r}_{I}(X)=X$ or $X=\overline{\operatorname{Apr}}_{I}(X)$.

(4) If $X \subseteq Y$, then $\underline{A p r}_{I}(X) \subseteq \frac{A p r}{I}(Y)$ and $\overline{A p r}_{I}(X) \subseteq \overline{A p r}_{I}(Y)$.

(5) $\underline{A p r}_{I}(X)=\left(\overline{A p r}_{I}\left(X^{c}\right)\right)^{c}$ and $\overline{A p r}_{I}(X)=\left(\underline{A p r}_{I}\left(X^{c}\right)\right)^{c}$.

(6) $\underline{A p r}_{I}(X \cap Y)=\underline{A p r}_{I}(X) \cap \underline{A p r}_{I}(Y)$.

(7) $\overline{A p r}_{I}(X \cap Y) \subseteq \overline{A p r}_{I}(X) \cap \overline{A p r}_{I}(Y)$.

(8) $\operatorname{Apr}_{I}(X \cup Y) \supseteq \underline{A p r}_{I}(X) \cup \underline{A p r}_{I}(Y)$.

(9) $\overline{A p r}_{I}(X \cup Y)=\overline{A p r}_{I}(X) \cup \overline{A p r}_{I}(Y)$.

(10) $\operatorname{Apr}_{0}(X)=(\emptyset, A)$.

(11) $\operatorname{Apr}_{A}(X)=(X, A)$.

Proof. It is straightforward.

Proposition 3.2. Let $\left(\mathfrak{A} ; I_{1}\right)$ and $\left(\mathfrak{A} ; I_{2}\right)$ be two approximation spaces and $I_{1} \subseteq I_{2}$. Then for each subset $X$ of $A$ the following conditions are satisfied.

(1) $\operatorname{Apr}_{I_{2}}(X) \subseteq \frac{A p r}{I_{1}}(X)$.

(2) $\overline{A p r}_{I_{1}}(X) \subseteq \overline{A p r}_{I_{2}}(X)$.

Proof. It is straightforward by $a / I_{1} \subseteq a / I_{2}$, for each $a \in A$.

Corollary 3.3. Let $\left\{\left(\mathfrak{A} ; I_{j}\right)\right\}_{j \in J}$ be a non empty family of approximation spaces. Then for any subset $X$ of $A$ the following condition holds:

$$
\cup_{j \in J} \underline{A p r}_{I_{j}}(X) \subseteq \underline{A p r}_{\cap_{j \in J} I_{j}}(X) \subseteq \cap_{j \in J} \overline{A p r}_{I_{j}}(X)
$$


Proof. We have $\cap_{j \in J} I_{j} \subseteq I_{j}$, for each $j \in J$. Thus by Proposition $3.2(\mathbb{D})$ we obtain that $\underline{A p r}_{I_{j}}(X) \subseteq \underline{A p r} \cap_{j \in J} I_{j}(X)$, for each $j \in J$ and it implies that $\cup_{j \in J} \underline{A p r}_{I_{j}}(X) \subseteq \underline{A p r}_{\cap_{j \in J} I_{j}}(X)$. Similarly, we can show that $\overline{A p r}_{\cap_{j \in J} I_{j}}(X) \subseteq \cap_{j \in J} \overline{A p r}_{I_{j}}(X)$ and it holds the result.

Proposition 3.4. Let $\left\{\left(\mathfrak{A} ; I_{i}\right)\right\}_{i \in I}$ be a non empty family of approximation spaces. Then for any subset $X$ of $A$ we have

$$
{\stackrel{A p r}{ } \vee_{i \in I} I_{i}}(X) \subseteq \cap_{i \in I} \underline{A p r}_{I_{i}}(X) .
$$

Proof. We know that $I_{i} \subseteq \vee_{i \in I} I_{i}$, for each $i \in I$. So by Proposition $\mathbf{2} 2(\mathbb{U})$, we conclude that $\underline{A p r} \vee_{i \in I} I_{i}(X) \subseteq \underline{A p r} I_{I_{i}}(X)$, for each $i \in I$ and it holds the result.

Proposition 3.5. Let $(\mathfrak{A} ; I)$ be an approximation space. Then for any non empty subset $X$ of $A$ the following assertions hold:

(1) $X \subseteq I$ if and only if $\overline{A p r}_{I}(X)=I$.

(2) $I \subseteq X$ if and only if $I \subseteq \underline{A p r}_{I}(X)$.

Proof.

Let $X \subseteq I$ and $a \in \overline{A p r}_{I}(X)$. Hence, there is $x \in X$ such that $(a, x) \in I^{\delta}$. Since, $X \subseteq I$ we obtain that $a \in I$, that is, $\overline{A p r}_{I}(X) \subseteq I$. Conversely, let $a \in I$. Then $a / I \cap X=X \neq \emptyset$. Therefore, $a \in \overline{A p r}_{I}(X)$, and hence $\overline{A p r}_{I}(X)=I$. The converse follows from Proposition उ.D(प).

Let $a \in I$. Therefore, $a / I=I \subseteq X$ and it shows that $I \subseteq \underline{A p r}_{I}(X)$. Conversely, if $a \in I$ then $I=a / I \subseteq X$.

Proposition 3.6. Let $(\mathfrak{A} ; I)$ be an approximation space and $J$ be an ideal of $\mathfrak{A}$. Then the following assertions hold:

(1) $I \subseteq J$ if and only if $\underline{A p r}_{I}(J)=J$.

(2) $I \subseteq \overline{\operatorname{Apr}}_{I}(J)$.

Proof.

Let $I \subseteq J$. By Proposition $3 . \mathbb{( \mathbb { U }})$, we have $\underline{A p r}_{I}(J) \subseteq J \subseteq \overline{A p r}_{I}(J)$. Now, let $a \in \overline{A p r}_{I}(J)$. So $a / I \cap J \neq \emptyset$ and it implies that there is $b \in J$ such that $(a, b) \in I^{\delta} \subseteq J^{\delta}$. Therefore,

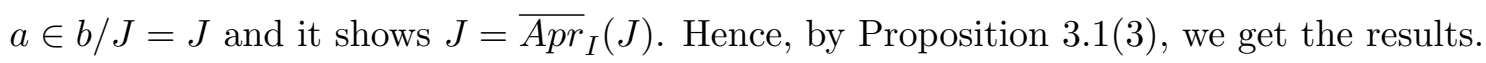

Conversely, suppose that $\underline{A p r}_{I}(J)=J=\overline{A p r}_{I}(J)$. If $a \in I$ then $0 \in a / I \cap J$ and it means that $a \in \overline{\operatorname{Apr}}_{I}(J)=J$.

Let $a \in I$. Then we have $0 \in a / I \cap J$ and it shows that $a \in \overline{\operatorname{Apr}}_{I}(J)$. 
Proposition 3.7. Let $h: \mathfrak{A} \longrightarrow \mathfrak{B}$ be a homomorphism. Then for any subset $X$ of $A$ we have

$$
h\left(\overline{\operatorname{Apr}}_{\mathrm{ker}(h)}(X)\right)=h(X) .
$$

Proof. According to Proposition B.](四), it is obvious that $h(X) \subseteq h\left(\overline{A p r}_{\operatorname{ker}(h)}(X)\right)$. Conversely, if $b \in h\left(\overline{A p r}_{\operatorname{ker}(h)}(X)\right)$ then exists $a \in \overline{A p r}_{\operatorname{ker}(h)}(X)$ such that $b=h(a)$. It shows that exists $x \in X$ such that $(a, x) \in \operatorname{ker}^{\delta_{\mathfrak{A}}}(h)=\kappa(h)$, by Proposition [.10. So $b=h(a)=h(x)$ and it shows that $b \in h(X)$.

Proposition 3.8. Let $h: \mathfrak{A} \longrightarrow \mathfrak{B}$ be an epimorphism and $I$ be an ideal of $\mathfrak{A}$ containing $\operatorname{ker}(h)$. Then for each subset $X$ of $A$ the following conditions hold:

(1) $h\left(\underline{A p r}_{I}(X)\right) \subseteq \underline{A p r}_{h(I)}(h(X))$.

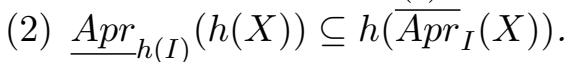

(3) $h\left(\overline{A p r}_{I}(X)\right) \subseteq \overline{A p r}_{h(I)}(h(X))$.

Proof.

Let $b \in h\left(\underline{A p r}_{I}(X)\right)$. So there is $a \in \underline{A p r}_{I}(X)$ such that $b=h(a)$. We have $h(a / I) \subseteq h(X)$ and by Lemma 2.15 we obtain that $b=h(a) \in \underline{A p r}_{h(I)}(h(X))$.

Let $b \in \underline{A p r}_{h(I)}(h(X))$. Therefore, $b / h(I) \subseteq h(X)$ and it implies that there exists $x \in X$ such that $b=h(x)$. Also, we have $x / I \cap X \neq \emptyset$ and it shows that $b \in h\left(\overline{A p r}_{I}(X)\right)$.

Let $b \in h\left(\overline{A p r}_{I}(X)\right)$. Hence, there exists $a \in \overline{A p r}_{I}(X)$ such that $b=h(a)$. We have $a / I \cap X \neq \emptyset$ and by using Lemma 2.15 it implies that

$$
\emptyset \neq h(a / I \cap X) \subseteq h(a / I) \cap h(X)=h(a) / h(I) \cap h(X) .
$$

Hence, $b=h(a) \in \overline{A p r}_{h(I)}(h(X))$.

Let $\mathcal{K}$ be an ideal determined class and $I$ be an ideal of $\mathfrak{A} \in \mathcal{K}$. The mapping $\pi_{I}^{\mathfrak{A}}: A \longrightarrow A / I$ defined by $\pi_{I}^{\mathfrak{A}}(a)=a / I$ is called the natural homomorphism. It is obvious that the natural map $\pi_{I}^{\mathfrak{A}}$ is an epimorphism and $\operatorname{ker}\left(\pi_{I}^{\mathfrak{A}}\right)=I$. If $X$ is a subset of $A$ then $\pi_{I}^{\mathfrak{A}}(X)=X / I$.

Corollary 3.9. Let $\left(\mathfrak{A}, I_{1}\right)$ and $\left(\mathfrak{A}, I_{2}\right)$ be two approximation spaces and $I_{1} \subseteq I_{2}$. Then for each subset $X$ of $A$ the following conditions hold:

(1) $\operatorname{Apr}_{I_{2}}(X) / I_{1} \subseteq \frac{A p r}{I_{2} / I_{1}}\left(X / I_{1}\right)$.

(2) $\operatorname{Apr}_{I_{2} / I_{1}}\left(X / I_{1}\right) \subseteq \overline{A p r}_{I_{2}}(X) / I_{1}$.

(3) $\overline{A p r}_{I_{2}}(X) / I_{1} \subseteq \overline{A p r}_{I_{2} / I_{1}}\left(X / I_{1}\right)$.

Proof. It is enough to consider the natural homomorphism $\pi_{I_{1}}^{\mathfrak{A}}$ in Proposition 3.8. 
Proposition 3.10. Let $h: \mathfrak{A} \longrightarrow \mathfrak{B}$ be a homomorphism and $I$ be an ideal of $\mathfrak{B}$. Then for each subset $X$ of $A$ and subset $Y$ of $B$ the following conditions are satisfied:

(1) $\left.h^{\leftarrow} \underline{A p r}_{I}(Y)\right) \subseteq \underline{A p r}_{h^{\leftarrow(I)}}\left(h^{\leftarrow}(Y)\right)$. Moreover, the equality holds if $h$ is surjective.

(2) $\operatorname{Apr}_{h^{\leftarrow(I)}}\left(h^{\leftarrow}(Y)\right) \subseteq h^{\leftarrow}\left(\overline{A p r}_{I}(Y)\right)$.

(3) If $h$ is an epimorphism then $h^{\leftarrow}\left(\overline{A p r}_{I}(Y)\right) \subseteq \overline{A p r}_{h^{\leftarrow}(I)}\left(h^{\leftarrow}(Y)\right)$.

(4) $\overline{A p r}_{h^{\leftarrow}(I)}(X) \subseteq h^{\leftarrow}\left(\overline{A p r}_{I}(h(X))\right)$.

Proof.

Let $h^{\leftarrow}\left(\underline{A p r}_{I}(Y)\right)$ be a non-empty set. Consider, $a \in f^{\leftarrow}\left(\underline{A p r}_{I}(Y)\right)$. Thus $h(a) \in \underline{A p r}_{I}(Y)$ and it means that $h(a) / I \subseteq Y$. By Lemma 2.16 we have $h\left(a / h^{\leftarrow}(I)\right) \subseteq Y$ and it shows that $a / h^{\leftarrow}(I) \subseteq h^{\leftarrow}(Y)$. Therefore, we obtain that $a \in \underline{A p r}_{h^{\leftarrow}(I)}\left(h^{\leftarrow}(Y)\right)$. Now, let $h$ be a surjection. By Proposition B.8(四) we have $h\left(\underline{A p r}_{h^{\leftarrow}(I)}\left(h^{\leftarrow}(Y)\right)\right) \subseteq \underline{A p r}_{h\left(h^{\leftarrow}(I)\right)}\left(h\left(h^{\leftarrow}(Y)\right)\right)=$ ${ }_{A p r}{ }_{I}(Y)$ and it shows that the equality holds.

Let $\underline{A p r}_{h^{\leftarrow(I)}}\left(h^{\leftarrow}(Y)\right)$ be a non-empty set. Let $a \in \underline{A p r}_{h^{\leftarrow}(I)}\left(h^{\leftarrow}(Y)\right)$. So $a / h^{\leftarrow}(I) \subseteq h^{\leftarrow}(Y)$ and it concludes that $h\left(a / h^{\leftarrow}(I)\right) \subseteq Y \cap h(a) / I$. Thus $a \in h^{\leftarrow}\left(\overline{A p r}_{I}(Y)\right)$.

Let $h$ be a surjection and $a \in h^{\leftarrow}\left(\overline{A p r}_{I}(Y)\right)$. So $h(a) \in \overline{A p r}_{I}(Y)$ and it concludes that $h(a) / I \cap Y \neq \emptyset$. Thus we have $h^{\leftarrow}(h(a) / I) \cap h^{\leftarrow}(Y) \neq \emptyset$ and it implies that $a / h^{\leftarrow}(I) \cap h^{\leftarrow}(Y) \neq$ $\emptyset$. It shows that $a \in \overline{A p r}_{h^{\leftarrow}(I)}\left(h^{\leftarrow}(Y)\right)$.

Let $a \in \overline{A p r}_{h^{\leftarrow}(I)}(X)$. So $a / h^{\leftarrow}(I) \cap X \neq \emptyset$ and it implies that $h(a) / I \cap h(X) \neq \emptyset$. It shows that $a \in h^{\leftarrow}\left(\overline{A p r}_{I}(h(X))\right)$.

\section{Rough Subalgebras AND ROUgh ideals}

In this section, we introduce the notion of rough subalgebras and rough ideals of an algebraic structure in an ideal determined variety as a generalization of the notion of subalgebras and ideals.

Definition 4.1. Let $\mathfrak{A}$ be an algebraic structure in $\mathcal{V}$ and $I$ be an ideal of $\mathfrak{A}$. A nonempty subset $X$ of $A$ is called an upper (resp., a lower) rough subalgebra (or ideal) of $\mathfrak{A}$ w.r.t $I$, if the upper (resp., the lower) approximation of $X$ w.r.t $I$ is a subalgebra (or an ideal ) of $\mathfrak{A}$. If $X$ is both an upper and a lower rough subalgebra (or ideal) of $\mathfrak{A}$ w.r.t $I$, we say $X$ is a rough subalgebra (or ideal) of $\mathfrak{A}$ w.r.t $I$.

Proposition 4.2. Let $\mathfrak{A}$ be an algebraic structure in $\mathcal{V}$ and $I$ be an ideal of $\mathfrak{A}$. If $S$ is a subalgebra of $\mathfrak{A}$ then $S$ is an upper rough subalgebra of $\mathfrak{A}$ w.r.t I.

Proof. Let $S$ be a subalgebra of $\mathfrak{A}, \sigma \in \Sigma$ and $a_{1}^{\rho(\sigma)} \in \overline{\operatorname{Apr}}_{I}(S)$. So there are $s_{i} \in S \cap a_{i} / I$ for each $1 \leq i \leq \rho(\sigma)$ such that $\left(a_{i}, s_{i}\right) \in I^{\delta}$. Since $I^{\delta}$ is a congruence relation then we 
have $\left(\sigma^{\mathfrak{A}}\left(a_{1}^{\rho(\sigma)}\right), \sigma^{\mathfrak{A}}\left(s_{1}^{\rho(\sigma)}\right)\right) \in I^{\delta}$. Therefore, $\left.\sigma^{\mathfrak{A}}\left(s_{1}^{\rho(\sigma)}\right)\right) \in S \cap \sigma^{\mathfrak{A}}\left(a_{1}^{\rho(\sigma)}\right) / I$ and it shows that $\sigma^{\mathfrak{A}}\left(a_{1}^{\rho(\sigma)}\right) \in \overline{\operatorname{Apr}}_{I}(S)$.

Corollary 4.3. Let $\mathfrak{A}$ be an algebraic structure in $\mathcal{V}$ and $I$ be an ideal of $\mathfrak{A}$. Then for each non empty subset of $X$ of $A$ we obtain

$$
S g\left(\overline{A p r}_{I}(X)\right) \subseteq \overline{A p r}_{I}(S g(X))
$$

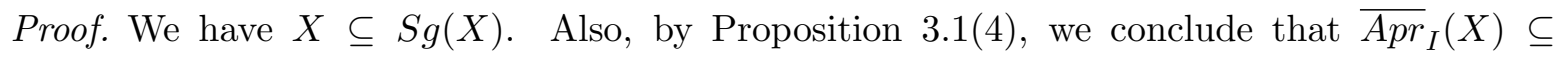
$\overline{A p r}_{I}(S g(X))$. By Proposition 4.2, we know that $\overline{A p r}_{I}(S g(X))$ is a subalgebra of $\mathfrak{A}$ and it means that $S g\left(\overline{A p r}_{F}(X)\right) \subseteq \overline{A p r}_{F}(S g(X))$.

Proposition 4.4. (Third Isomorphism Theorem) Let $\mathfrak{A}$ be an algebraic structure in $\mathcal{V}$ and I be an ideal of $\mathfrak{A}$. Then for any subalgebra $S$ of $\mathfrak{A}$ we have

$$
S / I \cap S \cong \overline{A p r}_{I}(S) / I \cap \overline{A p r}_{I}(S)
$$

Proof. By Lemma [.4, $I \cap S$ is an ideal of $S$ and $I \cap \overline{A p r}_{I}(S)$ is an ideal of $\overline{A p r}_{I}(S)$. We define the map $h: S \longrightarrow \overline{A p r}_{I}(S) / I \cap \overline{A p r}_{I}(S)$ by $h(s)=s / I \cap \overline{A p r}_{I}(S)$, for any $s \in S$. It is easy to check that $h$ is a homomorphism of residuated lattices. Consider $a / I \cap \overline{A p r}_{I}(S) \in \overline{A p r}_{I}(S) / I \cap$ $\overline{A p r}_{I}(S)$. So there is $s \in S$ such that $a / I=s / I$. One can check that $h(s)=a / I \cap \overline{A p r}_{I}(S)$ and it implies that $h$ is an epimorphism. So by the first isomorphism theorem we have

$$
S / \operatorname{ker}(h) \cong \overline{A p r}_{I}(S) / I \cap \overline{A p r}_{I}(S) .
$$

If $s \in \operatorname{ker}(h)$ then $s / I \cap \overline{A p r}_{I}(S)=0 / I \cap \overline{A p r}_{I}(S)$. Since, $\mathcal{V}$ is a variety it implies that $d_{i}(s, 0) \in\left(I \cap \overline{A p r}_{I}(S)\right)^{\delta_{S}}$, for each $1 \leq i \leq n$. Thus $\left.s \in I \cap \overline{A p r}_{I}(S)\right)$ and it means $s \in I$. Therefore, $\operatorname{ker}(h) \subseteq I \cap S$. On the other hand, $I \cap S \subseteq \operatorname{ker}(h)$ and it shows that $\operatorname{ker}(h)=I \cap S$.

Proposition 4.5. Let $\mathfrak{A}$ be an algebraic structure in $\mathcal{V}$ and $I$ be an ideal of $\mathfrak{A}$. Then for any ideal $J$ of $\mathfrak{A}, \overline{A p r}_{I}(J)$ is an ideal of $\mathfrak{A}$.

Proof. Let $\alpha\left(t_{1}^{n}, s_{1}^{m}\right)$ be an ideal term, $a_{1}^{n} \in A$ and $b_{1}^{m} \in \overline{A p r}_{I}(J)$. Hence, there are $j_{1}^{m} \in J$ such that $\left(b_{i}, j_{i}\right) \in I^{\delta}$, for each $1 \leq i \leq m$. It implies that $\left(\alpha\left(a_{1}^{n}, b_{1}^{m}\right), \alpha\left(a_{1}^{n}, j_{1}^{m}\right)\right) \in I^{\delta}$ and so $\left.\alpha\left(a_{1}^{n}, j_{1}^{m}\right) \in \alpha\left(a_{1}^{n}, b_{1}^{m}\right)\right) / I^{\delta}$. On the other hand, since $J$ is an ideal of $\mathfrak{A}$ then $\alpha\left(a_{1}^{n}, j_{1}^{m}\right) \in J$ and it shows that $\left.\alpha\left(a_{1}^{n}, b_{1}^{m}\right)\right) / I^{\delta} \cap J \neq \emptyset$. Therefore, $\left.\alpha\left(a_{1}^{n}, b_{1}^{m}\right)\right) \in \overline{A p r}_{I}(J)$ and it results $\overline{A p r}_{I}(J)$ is an ideal of $\mathfrak{A}$. 
Alg. Struc. Appl. Vol. 6 No. 1 (2019) 1-21.

Corollary 4.6. Let $\mathfrak{A}$ be an algebraic structure in $\mathcal{V}$ and $I$ be an ideal of $\mathfrak{A}$. Then for each non empty subset $X$ of $A$ we have

$$
\operatorname{Id}\left(\overline{\operatorname{Apr}}_{I}(X)\right) \subseteq \overline{\operatorname{Apr}}_{I}(\operatorname{Id}(X)) .
$$

Proof. We have $X \subseteq I d(X)$. Also, by Proposition $[].(\mathbb{U})$, we conclude that $\overline{A p r}_{I}(X) \subseteq$ $\overline{A p r}_{I}(\operatorname{Id}(X))$. By Proposition 4.5 , we know that $\overline{A p r}_{I}(\operatorname{Id}(X))$ is an ideal of $\mathfrak{A}$ and it shows that $\operatorname{Id}\left(\overline{A p r}_{I}(X)\right) \subseteq \overline{A p r}_{I}(I d(X))$.

\section{ACKNOWledgments}

The author wish to sincerely thank the referees for several useful comments.

\section{REFERENCES}

[1] P. Agliano and A. Ursini, Ideals and other generalizations of congruence classes, J. Aust. Math. Soc. Ser. A 53 (1992), 103-115

[2] P. Agliano and A. Ursini, On subtractive varieties II: General properties, Algebra Universalis 36 (1996), $222-259$.

[3] R. Biswas and S. Nanda, Rough groups and rough subgroups, Bull. Polish Acad. Sci. Math. 42 (1994), $251-254$.

[4] R. Belohlávek and I. chajda, Congruence classes in regular varieties, Acta Math. Univ. Comenianae 68(1) (1999), 71-75.

[5] Z. Bonikowski, Algebraic structures of rough sets, Rough sets, fuzzy sets and Knowledge discovery. Springer, London, (1994), 242-247.

[6] S. Burris and H. A. Sankappanavar, A Course in Universal Algebra, Springer, Berlin, 1981.

[7] B. Davvaz, Roughness in rings, Inf. Sci. 164 (2004) 147-163.

[8] B. Davvaz, A short note on algebraic T-rough sets, Inf. Sci. 178 (2008) 3247-3252.

[9] B. Davvaz and M. Mahdavipour, Roughness in modules, Inf. Sci. 176 (2006) 3658-3674.

[10] K. Denecke, M. Erné and S. L. Wismath, Galois connections and applications, volume 565. Springer, 2004.

[11] D. Dubois and H. Prade, Rough fuzzy sets and fuzzy rough sets, International Journal of General Systems 17 (1990), 191-209.

[12] F. Feng, C. Li, B. Davvaz and M.I. Ali, Soft sets combined with fuzzy sets and rough sets: a tentative approach, Soft Computing 14 (2010), 899-911.

[13] K. Fichtner, Varieties of universal algebras with ideals, Mat. Sbornik 75(117) (1968), 445-453. (In Russian.)

[14] K. Fichtner, Eine Bermerkung ber Mannigfaltigkeiten universeller Algebren mit Idealen, Monatsh. d. Deutsch. Akad. d. Wiss. (Berlin) 12 (1970), 21-25.

[15] B. Ganter and R. Wille, Formal Concept Analysis, Mathematical Foundations, Springer, Berlin, 1999.

[16] G. Gediga, I. Düntsch, Modal-style operators in qualitative data analysis, in: Proceedings of the 2002 IEEE International Conference in Data Mining (2002), 155-162.

[17] H.P. Gumm and A. Ursini, Ideals in universal algebras, Algebra Universalis 19 (1984), 45-55. 
[18] F. García Pardo, I. P. Cabrera, P. Cordero and M. Ojeda-Aciego, On Galois connections and soft computing, Lecture Notes in Computer Science, (2013) 224-235.

[19] G. Grätzer, Universal Algebra, Van Nostrand, Princeton, N.J., 1968.

[20] P. J. Higgins, Groups with multiple operators, Proc. London Math. Soc. 3(3) (1956), 366-416.

[21] M. Irfan Ali, B. Davvaz and M. Shabir, Some properties of generalized rough sets, Inf. Sci. 224 (2013), 170-179.

[22] T. Iwinski, Algebraic approach to rough sets, Bull. Pol. Acad. Sci. Math. 35 (1987) 673683.

[23] W. Krull, Axiomatische Begrundung der allgemeinen Idealtheorie, Sitzungsberichteder Physikalisch Medizinischen Societatder Erlangen 56 (1924), 47-63.

[24] N. Kuroki and P.P. Wang, The lower and upper approximations in a fuzzy group, Inf. Sci. 90 (1996), $203-220$.

[25] N. Kuroki, Rough ideals in semigroups, Inf. Sci. 100 (1997), 139-163.

[26] H. Lai, D. Zhang, Concept lattices of fuzzy contexts: Formal concept analysis vs. rough set theory, International Journal of Approximate Reasoning 50 (2009), 695-707.

[27] R. Magari, Su una classe equazionale di algebre, Ann. Mat. Pura Appl. 75(1) (1967), 277-312.

[28] Z. Pawlak, Rough sets, Int. J. Inf. Comput. Sci. 11 (1982), 341-356.

[29] J. Pomykala and J. A. Pomykala, The stone algebra of rough sets, Bull. Pol. Acad. Sci. Math. 36 (1998), $495-508$.

[30] S. Rasouli, Heyting Boolean and pseudo-MV filters in residuated lattices, Journal of Multiple Valued Logic and Soft Computing 31(4) (2018), 287-322.

[31] S. Rasouli and B. Davvaz, Lattices derived from hyperlattices, Communications in Algebra@ $38(8)(2010)$, $2720-2737$

[32] S. Rasouli and B. Davvaz, $\beta$-relations on implicative bounded hyper BCK-algebras, Hacettepe Journal of Mathematics and Statistics 39(4) (2010), 461-469.

[33] S. Rasouli and B. Davvaz, Roughness in MV-algebras, Inf. Sci. 180(5) (2010), 737-747.

[34] S. Rasouli and B. Davvaz, Homomorphism, Ideals and Binary Relations on Hyper-MV Algebras, Multiplevalued Logic and Soft Computing 17(1) (2011), 47-68.

[35] S. Rasouli, B. Davvaz, An Investigation on Algebraic Structure of Soft Sets and Soft Filters over Residuated Lattices, ISRN Algebra, vol. 2014, Article ID 635783, 8 pages, 2014. doi:10.1155/2014/635783.

[36] S. Rasouli and B. Davvaz, An investigation on Boolean prime filters in BL-algebras, Soft Computing 19(10) (2015), 2743-2750.

[37] S. Rasouli and B. Davvaz, An investigation on regular relations of universal hyperalgebras, Algebraic Structures and Their Applications 5(1) (2018), 1-21.

[38] S. Rasouli and B. Davvaz, Rough filters based on residuated lattices, Knowledge and Information Systems 58(2) (2018), 399-424.

[39] S. Rasouli and A. Radfar, PMTL filters, Rl filters and PBL filters in residuated lattices, Journal of Multiple Valued Logic and Soft Computing 29(6) (2017), 551-576.

[40] S. Rasouli, Z. Zarin and A. Hassankhani, Characterization of a new subquasivariety of residuated Lattice, Journal of applied logics-The IfCoLog journal of logics and their applications 5(1) (2018), 33-63. 
Alg. Struc. Appl. Vol. 6 No. 1 (2019) 1-21.

[41] A. Ursini, Sulle varietà di algebre con una buona teoria degli ideali, Boll. Unione Mat. Ital. 6(4) (1972), 90-95.

[42] A. Ursini, Osservazioni sulle varietà BIT, Boll. Unione Mat. Ital. 7(4) (1973), 205-211.

[43] A. Ursini, On subtractive varieties I, Algebra Universalis 31 (1994), 204-222.

[44] R. Wille, Restructuring lattice theory: An approach based on hierarchies of concepts, Ordered Sets, I. Rival (Ed.), Reidel, (1982), 445-470.

[45] Q. Xiaoa, Q. Lia and L. Guo, Rough sets induced by ideals in lattices, Inf. Sci. 271 (2014), 82-92.

[46] S. Yamaka, O. Kazancia and B. Davvaz, Generalized lower and upper approximations in a ring, Inf. Sci. 180 (2010) 1759-1768.

[47] Y.Y. Yao, Constructive and algebraic methods of the theory of rough sets, Inf. Sci. 109 (1998), 21-47.

\section{Saeed Rasouli}

Department of Mathematics, College of science, Persian Gulf University,

Bushehr, 7516913817, Iran

srasouli@pgu.ac.ir 